\title{
On Optimizing Autonomous Pipeline Inspection
}

\author{
Xin Li, Member, IEEE, Wuyi Yu, Student Member, IEEE, Xiao Lin, and S. S. Iyengar, Fellow, IEEE
}

\begin{abstract}
This paper studies the optimal inspection of autonomous robots in a complex pipeline system. We solve a 3-D region-guarding problem to suggest the necessary inspection spots. The proposed hierarchical integer linear programming optimization algorithm seeks the fewest spots necessary to cover the entire given 3-D region. Unlike most existing pipeline inspection systems that focus on designing mobility and control of the explore robots, this paper focuses on global planning of the thorough and automatic inspection of a complex environment. We demonstrate the efficacy of the computation framework using a simulated environment, where scanned pipelines and existing leaks, clogs, and deformation can be thoroughly detected by an autonomous prototype robot.
\end{abstract}

Index Terms-Autonomous pipeline inspection, 3-D region guarding.

\section{INTRODUCTION}

A CTIVE monitoring and frequent inspections are critical to maintaining pipeline health. As the most economical way to transport gas, oil, bio fuels, water resource, sewer, and so forth, pipelines have become an indispensable part of our daily lives. However, pipelines always suffer from aging and damages, which can cause great waste of resource, environmental pollution, and many other accidence. For example, the leak of petroleum pipeline causes ocean pollution and ecocatastrophe. Regular inspections and maintenance of pipelines are essential to keep them functional.

Unfortunately, the difficulty and the cost for human inspection can be extremely high, especially with the appearance of increasingly complicated pipelines nowadays (see, for example, Fig. 1). There are several reasons.

1) Pipeline systems are often buried/hided underground or into walls. Hiding pipelines' presence from the surround-

Manuscript received November 14, 2010; revised May 5, 2011; accepted September 16, 2011. Date of publication November 1, 2011; date of current version February 9, 2012. This paper was recommended for publication by Associate Editor T. Murphey and Editor J.-P. Laumond upon evaluation of the reviewers' comments. This work was supported in part by the National Natural Science Foundation of China under Grant 61170323, the U.S. National Science Foundation under Grant CNS-1158701, the Louisiana (LA) Post Katrina funds, the LA Board of Regents RCS LEQSF(2009-12)-RD-A-06, and PFund: NSF(2011)-PFund-236.

$\mathrm{X}$. Li is with the Department of Electrical and Computer Engineering, and Center for Computation and Technology, Louisiana State University, Baton Rouge, LA 70803 USA (e-mail: xinli@ cct.lsu.edu).

W. Yu and X. Lin are with the Department of Automation, Xiamen University, Xiamen 361000, China (e-mail: yuwuyi@gmail.com; 1xsherryxmu@ gmail.com).

S. S. Iyengar is with the School of Computing and Information Sciences, Florida International University, Miami, FL 33199 USA (e-mail: iyengar@ cis.fiu.edu).

This paper has supplementary downloadable material available at http://ieeexplore.ieee.org, provided by the authors.

Color versions of one or more of the figures in this paper are available online at http://ieeexplore.ieee.org.

Digital Object Identifier 10.1109/TRO.2011.2169619

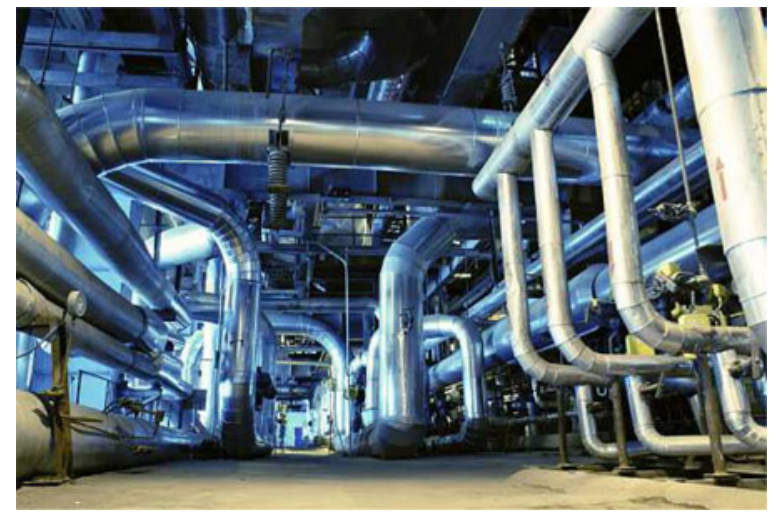

Fig. 1. Complex pipeline system. Image from [1].

ing environment is necessary for better protection of pipelines, as well as the elegance of the architecture.

2) The structure of pipelines is usually designed long, thin, and complex, in order to conduct long-distance transportation or circumvent-complicated terrain or limited space of the architecture structure.

3) The environment inside pipelines can be dirty and hazardous: Sewerage water or hazardous gas can be overflowing.

These factors make direct artificial inspection oftentimes prohibitive, and, therefore, significantly increase the costs for the maintenance of pipelines. For example, to inspect the pipelines, people can dig holes in different pipeline sections for the inspection; high professional competence could be necessary especially when the environment condition is severe. Indirect methods include placing sensors outside the pipeline and monitoring parameters such as pressure, temperature, etc. However, the sensibility is easily affected by environment and the material transmitting in the pipeline. Pipeline clogs are sometimes directly penetrated with long sticks or wires, but it can be very difficult if pipes are curved or circumvented; another common approach is to blow out blockages using air pressure, which fails, however, if pipes have multiple outlets or cracks. In any case, to apply repair, the suspected area for clogging or leaking need to be located. This step usually takes the longest time and largest cost.

\section{A. Pipeline Inspection by Autonomous Robots}

With the development of autonomous robots and imaginarysensing technologies, pipeline robots that are equipped with cameras and sensors become ideal candidates to avoid tedious artificial inspection for automatic pipeline inspection and repair.

Current robotic inspection systems (see Section II) usually have a robot that is equipped with a camera and sensors; the robot moves around and transmits the captured images back to a remote monitor for the operator's inspection. The robot's 
movement and the camera direction need to be manually controlled by a skillful inspector. Such an interactive monitoring system provides easier and safer inspection. However, it can still be labor intensive (costly) and time consuming. In addition, complex pipeline environments could limit the extensive use of these remotely controlled pipeline robots. The mobility of remotely controlled robots could not meet the requirement for the inspection in complex pipeline environments, such as the urban gas pipeline, chemical pipelines, etc. The wired connections also limit the operation range of robots. Furthermore, the thoroughness of the examination may not be guaranteed and heavily relies on expertise of the operator.

An autonomous robot that can routinely inspect the environment and report cracks, clogs, or deformation will, therefore, be highly desirable. If such an inspection system can be developed reliably and conducted routinely, it will greatly save artificial costs and prevent the abnormal situations in important pipelines. To develop an efficient inspection plan for robots, according to different environments, to ensure inspection reliability (thoroughness) is related to several challenging geometric problems.

\section{B. Optimal Autonomous Inspection by Region Guarding}

Naturally, one wants to ask the following fundamental open problem for autonomous inspection: How do we conduct the most efficient yet thorough inspection? More specifically, given an environment to inspect, how many inspection spots are necessary to visually cover the entire region? The solution directly dictates the correctness and efficiency of an autonomous inspection system and, therefore, is critical.

Visually covering a given 3-D region is an interesting geometric problem called gallery guarding, defined (see Section III for details) as follows: Given a region whose boundary is a surface, find the smallest set of points inside the region from which all the boundary points (i.e., the wall) are visible. This problem, having high complexity, has been actively studied in 2-D. On general 2-D polygonal regions, this problem has been proved to be NP-hard. To the best of our knowledge, on 3-D regions approximated by polyhedra, which have much higher complexity, this problem is little explored and no efficient algorithm has been reported.

In this paper, we design a hierarchical integer linear programming (HILP) algorithm to find an approximate optimal solution for the guarding of a given 3-D region. Compared with the greedy and the optimal algorithms, HILP has a good approximation to the optimal solution (for instance, to guard a region in Fig. 3(d), an optimal guarding needs 13 guards, the greedy approach needs 18 points, while our HILP algorithm guards it with 14 points) but is several orders of magnitude less than the direct optimization on time complexity.

Effective region guarding can greatly benefit the automatic pipeline inspection. Compared with existing manual inspection systems, the biggest advantage of the new inspection system built upon optimal guarding is its thorough (therefore, making the system robust) inspection using fewest (therefore, making the system efficient and inexpensive) necessary checking spots.
PIPELINE GUARDING/INSPECTION CHART

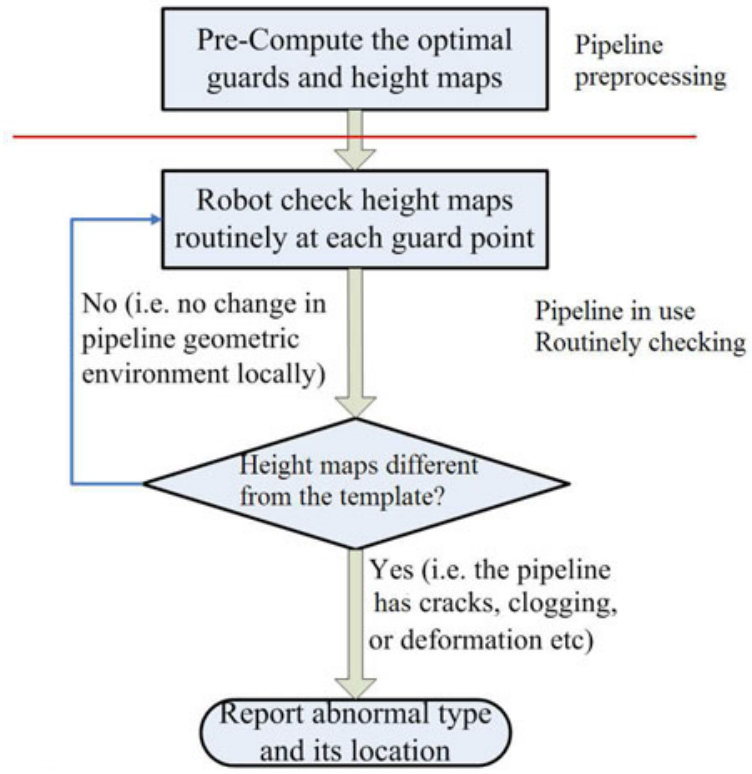

Fig. 2. Autonomous inspection based on region guarding.

The proposed inspection framework is illustrated in Fig. 2, and the pipeline has two steps.

1) Preprocessing Stage: Once pipeline is newly installed or when it is working well, we compute its optimal guarding, e.g., a small set of points $\left\{g_{i}\right\}$. By checking on these spots, the entire pipeline can be visually covered. Then, a pipeline robot only goes to these points, scans the pipe, and builds up sequential height maps as templates. These height maps characterize the original pipeline geometry.

2) Online Stage: The robot will go into the pipeline to conduct inspection routinely. Every time, it only needs to move to these spots $\left\{g_{i}\right\}$, scan the depth information of the surrounding environment, and compare these height maps with the corresponding templates. Abnormal geometry changes such as cracks, clogs, and deformation can be detected and located immediately.

The main contributions of this work lie in both efficiently finding good approximate solutions of the NP-hard 3-D guarding problem and its application on robotic inspection.

1) Optimality: We develop an efficient algorithm to find approximate solution to $3-\mathrm{D}$ region guarding. The solution indicates a smallest set of spots from which thorough inspection can be most timely and costly efficient.

2) Autonomy: We design an automatic pipeline inspection system for autonomous robots. Unlike other existing systems whose inspection quality heavily relies on manual controls, in our framework, robots can inspect on these fewest guarding points autonomously yet thoroughly.

3) Generality and Robustness: The algorithm efficacy is demonstrated in our simulated platform. It is also generally applicable on various robot systems, such as our pipe robot prototype FAMPER [2], which is equipped with a range sensor that provides $2.5-\mathrm{D}$ range image with depth 
TABLE I

UPPER BOUNDS FOR THE GALLERY GUARDING PROBLEM

\begin{tabular}{|c|c|c|c|}
\hline Work & $M$ Type & Guard Type & Optimal Bounds \\
\hline$[4]$ & simple, 2D & stationary & $\lfloor n / 3\rfloor$ \\
\hline$[5]$ & simple, 2D & mobile & $\lfloor n / 4\rfloor$ \\
\hline$[6]$ & simple, 2D & edge & seems $\lfloor n / 4\rfloor$ \\
\hline$[7],[8]$ & orthogonal, 2D & stationary & $\lfloor n / 4\rfloor$ \\
\hline$[9]$ & $h$ holes, 2D & vertex & $\left\lfloor\frac{n+2 h}{3}\right\rfloor$ \\
\hline$[10],[11]$ & $h$ holes, 2D & point & $\left\lfloor\frac{n+h}{3}\right\rfloor$ \\
\hline$[12]$ & orthogonal, $h$ holes, 2D & mobile & $\left\lfloor\frac{3 n+4 h+4}{16}\right\rfloor$ \\
\hline
\end{tabular}

information. Furthermore, combined with 2-D image reconstruction techniques, our algorithm can work well for robots that are equipped with a conventional 2-D camera.

\section{BACKGROUND AND RELATED WORK}

\section{A. Gallery Guarding}

On a geometric region $M$, we want to find the optimal guarding, which uses the smallest number of points $\left\{g_{i}\right\}$ inside $M$ so that any boundary point $p \in \partial M$ is visible to at least one guard. Here, $M$ is a 3-D shape whose boundary $\partial M$ is represented by a polygonal mesh. For a given guard $g_{i}$, any point $p \in \partial M$ is visible to $g_{i}$ if the line segment $\overline{g_{i} p}$ is entirely located inside $M$ (we consider $\partial M \subset M$ ). Various versions of this problem are generally called art-gallery problems, which are known to be a famous problem with high complexity. Even in the 2-D case, the problem is known to be NP-complete. "Very little is known about gallery guarding in three dimensions" [3]. To our best knowledge, no effective approximation algorithm has been proposed for 3-D regions that are bounded by general polygons, and this is the first practical algorithm that works for large free-form 3-D domains (such as complicated pipeline systems) represented by polygonal meshes.

The art-gallery problem was first proposed by Klee. Guards can be restricted to boundary vertices $(p \in \partial M)$, interior vertices $(p \in M)$, or mobile vertices. When guards are not mobile, they are called stationary guards. If guards are restricted to the boundary, they are called vertex guards; if there is no boundary restriction, the guards are referred as point guards. In 2-D, Chavatal [4] and Fisk [13] both showed that a simple polygon $M \subset \mathbb{R}^{2}$ needs at most $\lfloor n / 3\rfloor$ stationary guards, based on which, Avis and Toussaint [14] developed an $O(n \log n)$ time algorithm to position $\lfloor n / 3\rfloor$ guards in $M$. When guards are mobile, we call them mobile guards. Furthermore, mobile guards are called edge guards if they are restricted to boundary vertices. O'Rourke [5] showed that $\lfloor n / 4\rfloor$ mobile guards are sufficient for a simple polygon $M \subset \mathbb{R}^{2}$. More results are recapped in Table I.

The aforementioned theoretic work discusses the conservative upper bounds for necessary guards on various regions. Given a specific region, we are interested in designing practical algorithm to find its optimal point guards, which depends on topology and geometry of this region. An effective algorithm to compute the optimal guarding of a given region will benefit many geometric computing tasks. However, computation of optimal guarding is highly challenging. Finding minimal guards has been shown to be NP-hard for 2-D polygons with holes [15],
2-D simple polygons [16], and even 2-D simple orthogonal polygons [17], [18], using either vertex or point guards. Approximation algorithms have been studied in 2-D to get a closeto-optimal result in polynomial time complexity. Ben-Moshe et $a l$. [3] cover 1.5-D terrain using point guards in $O\left(n^{2}\right)$ time, with the optimal factor $O(1)$. Efrat and Har-Peled [19] find vertex guards for 2-D simple polygonal regions and $h$-hole polygonal regions in $O\left(n c_{\mathrm{opt}}^{2} \log ^{4} n\right)$ and $O\left(n c_{\mathrm{opt}}^{2} \log ^{4} n\right)$ expected time, with expected $O\left(\log c_{\mathrm{opt}}\right)$ and $O\left(n h c_{\mathrm{opt}}^{3} \log ^{4} n\right)$ optimal factors, respectively. Lien [20] computes guarding for 3-D point cloud data, approximating visibility using $\epsilon$-view. The algorithm is based on a randomized greedy approach.

\section{B. Pipeline Inspection Robots}

According to the degree of autonomy, pipeline inspection robots can be classified as follows [21].

1) No Autonomy: Robots are fully teleoperated by humans via a tether cable. While the robot is traveling through the pipe, the pipeline condition data are collected and sent back by the robot, and then assessed by human operators.

2) Semiautonomy: Robots are partially controlled by automatic control programs [22].

3) Full Autonomy: Robots are fully controlled by programs, and perform an automatic pipeline condition assessment. However, lacking effective technologies in efficient analysis of environment hinders the automatic assessment [21].

1) Manual and Semiautonomous Methods: There are many application inspection technologies, such as closed-circuit television (CCTV), laser surveys, sonar surveys [23], rediofrequency identification [24], and mobile sensor [25]. The introduction of CCTV inspection methods in the 1960s provided an inexpensive and safe option, and they, thus, have been the most popular and widely used approaches across the industry for many years. The CCTV provides rich videos/images information, which is collected by robots for subsequent pipeline condition assessment. Various CCTV methods have similar principles. The robot is mounted by a remotely controlled tractor, carries a television camera, and illuminates the interior of the pipe. The inspector has to identify and categorize defects by the image displayed on the monitor. When a defect is noticed, the inspector stops the robot and assesses the condition.

Advances in optical survey techniques have been utilized in the sewer scanner and evaluation technology (SSET) such as in [26]. Unlike the CCTV inspection system, the SSET may not need to stop for a zooming-in defect inspection. For instance, recent work in [27] has advanced the use of automated defect detection systems for pipelines.

Laser-based systems and ultrasonic-based systems are also used in pipeline inspection (see the survey in [28]). Laser-based systems are generally implemented in two ways: the whole circle image method and the single spot scanning method [29]. The first method projects a full ring of light onto the wall in one go, while the single spot scanning method sends point-bypoint beams in sequential. These two methods indicate a tradeoff between accuracy and inspection time. The whole-ring image method allows faster data acquisition but has been found less ac- 
curate [30]. Ultrasonic-based systems use high-frequency sound waves to detect pipe properties such as thickness, shape, and presence/sizes of defects [31]. Laser-based and ultrasonic-based methods can be combined to obtain higher quality data [32].

2) Autonomous Methods: A few full-autonomous robots have been developed for pipeline inspection. The Kurt [33] can run in dry clean pipelines guided by maps uploaded into the robot. The Marko [34] is designed for autonomous navigation in clean pipelines with diameter ranges from 300 to $600 \mathrm{~mm}$. The Kantaro [21] is used to navigate in pipelines with diameter ranges from 200 to $300 \mathrm{~mm}$, but only the horizontal mobility is considered. To design autonomous pipeline inspection robots, the main challenges include their moving ability, energy, and pipe condition assessment [35].

In this paper, we use laser range finders to detect the depth (height) information toward sets of sample directions. The captured 3-D range images provide easy measurement of the environment. We focus on designing the algorithm and architecture of the effective autonomous inspection system. The guarding and subsequent inspection can easily extend to various systems that are based on different data acquisition schemes.

\section{3-D GALLERY GUARDING}

The geometric abnormalities of the pipeline can be detected from the robot if the robot can see this region and measure the distance from itself to the pipe. Suppose the robot always checks at a set of same spots, and it has premeasured (template) distance information on each spot toward different directions, then it can tell whether the current pipeline is normal, i.e., preserving the same shape. These checking spots need to be intelligently selected so that fewest comparisons are necessary. Meanwhile, to guarantee that the entire pipeline is visually covered, we require that these points together can guard the entire region.

Given a point $p$ inside the region $M$, suppose we represent the boundary surface of $M$ using a triangle mesh $\partial M=$ $\{T, V\}$, where $V=\left\{v_{1}, v_{2}, \ldots, v_{N_{V}}\right\}$ is the vertex set, and $T=\left\{t_{1}, t_{2}, \ldots, t_{N_{T}}\right\}$ is the set of triangles connecting them. We say that $p$ is visible to a point $q$ on $\partial M(q$ is not necessary a vertex; it can be a point on a triangle from $T$ ), if the line segment $\overline{p q}$ connecting $p$ and $q$ is totally inside $M$, namely, $\overline{p q}$ intersects $\partial M$ only on $q$. We call the set of all visible points on the boundary $\{q\}, q \in \partial M$ the visible region $S(p)$ of $p$. Then, we say that a set of points $\{p\}$ can visibly guard the entire region, if the union of their visible regions is the entire $\partial M$. Finding a smallest guarding set $\{p\}$ that can cover the entire region is the optimal guarding problem that we want to solve. Our algorithm is based on the following intuitions.

1) As demonstrated in several medical visualization and virtual navigation applications (e.g., [36] and [37]), medial axes (curve skeletons) usually have desirable visibility to boundary points (referred as the "reliability" of skeletons). An effective skeleton can guide the camera navigation, ensuring nice examination (visibly covered) of the interior of organ surfaces.

2) Hierarchical skeletons or skeletons for a progressively simplified mesh can be effectively computed and used to reduce the size of the optimization problem, leading to a computation of better numerical efficiency and stability against boundary perturbations.

Many effective skeletonization algorithms (see a survey by Cornea et al. [38]) have been developed for 3-D shapes. We use the algorithm in [39] since it efficiently generates skeletons on medial-axis surfaces of the 3-D shapes. Suppose the boundary surface $\partial M$ of a volumetric region $M$ is represented by a triangle mesh (also denoted as $\partial M$ ) with $n$ vertices, and the output skeleton has $k$ nodes; the guarding problem is then converted to finding a minimal-size point set $G$ from this $k$ points, such that all $n$ boundary vertices are visible to $G$.

\section{A. Visibility Detection}

A basic operation is to detect the visible region $S(p)$ of a given point $p$. Following the definition, for a point $q \in \partial M$, to check its visibility to a point $p \in M$, one should check intersection between the line segment $\overline{p q}$ and $\partial M$. If the intersection is detected on a point $q^{\prime} \in \partial M$ other than $q$ and the Euclidean distance $\left|\overline{p q^{\prime}}\right|<|\overline{p q}|$, then $q$ is not visible from $p$. The intersection between the line segment $\overline{p q}$ and $\partial M$ can be detected by checking the intersection between $\overline{p q}$ and each triangle face $t_{i} \in T \subset \partial M$.

We need to compute the visibility of $p$ against all the vertices of the mesh of $\partial M$. Simply enumerating every $\overline{p v_{i}}$ to check its intersections with every triangle $t \in T$ is time consuming: For a single interior point $p$, it takes $O\left(N_{V} \cdot N_{T}\right)=O\left(N_{V}^{2}\right)$ time to check its visibility on all boundary vertices. We develop the following sweep algorithm to improve the efficiency to $O\left(N_{T} \log N_{T}\right)$, i.e., $O\left(N_{V} \log N_{V}\right)$.

We create a spherical coordinate system which is originated at $p$. Each vertex $v_{i} \in V$ is represented as $\overline{p v_{i}}=\left(r\left(v_{i}\right)\right.$, $\left.\theta\left(v_{i}\right), \varphi\left(v_{i}\right)\right)$, where $r\left(v_{i}\right) \geq 0,-\pi<\theta\left(v_{i}\right) \leq \pi, \frac{-\pi}{2} \leq \varphi\left(v_{i}\right)$ $\leq \frac{\pi}{2}$. For every triangle $t_{i}=\left(v_{i, 1}, v_{i, 2}, v_{i, 3}\right) \in T, 1 \leq i \leq n_{T}$, its $\max \theta\left(t_{i}\right)$ can be defined as $\theta_{\max }\left(t_{i}\right)=\max \left\{\theta\left(v_{i, j}\right)\right\}, 1 \leq$ $j \leq 3 ; \theta_{\min }\left(t_{i}\right), \varphi_{\max }\left(t_{i}\right)$, and $\varphi_{\min }\left(t_{i}\right)$ can be defined similarly.

The segment $\overline{p v_{k}}$ cannot intersect with a triangle $t$ unless they are adjacent:

$$
\left\{\begin{array}{l}
\theta_{\min }(t) \leq \theta\left(v_{k}\right) \leq \theta_{\max }(t) \\
\varphi_{\min }(t) \leq \varphi\left(v_{k}\right) \leq \varphi_{\max }(t) .
\end{array}\right.
$$

The angle functions $\theta$ and $\varphi$ are not continuously defined on a sphere. When a triangle $t$ spans $\theta=\pi$, we duplicate it to ensure that each $\theta$ of the original $t$ is between $\left[\theta_{\min }(t)-2 \pi, \theta_{\min }(t)\right)$ and $\theta$ of its duplicate is between $\left[\theta_{\max }(t), \theta_{\max }(t)+2 \pi\right)$, by adding or subtracting $\theta$ by $2 \pi$. For each triangle $t$ that spans $\varphi=\pi$, we detect and duplicate it in the same way. Using $\theta\left(v_{i}\right)$ as the primary key and $\varphi\left(v_{i}\right)$ as the secondary key, we then sort all line segments $\overline{p v_{i}}$. Then, we sweep all segments following the angle functions one by one, filtering out triangles not satisfying condition (1). Specifically, we define a counter $c_{i}$ on every triangle $t_{i}$. Initially, $c_{i}=0$; when the segment $\overline{p v}, v \in t_{i}$, is being processed, $c_{i} \leftarrow c_{i}+1$. The following two cases indicate that the sweep has not reached the neighborhood of the triangle $t_{i}$, and we do not need to check its intersection with line 
segment $\overline{p v}$

$$
\begin{aligned}
& c_{i}=0 \rightarrow \theta_{\min }\left(t_{i}\right), \quad \theta(\overline{o v}), \quad \text { or } \varphi_{\min }\left(t_{i}\right)>\varphi(\overline{o v}) \\
& c_{i}>3 \rightarrow \theta_{\max }\left(t_{i}\right)<\theta(\overline{o v}), \quad \text { or } \varphi_{\max }\left(t_{i}\right)<\varphi(\overline{o v}) . \quad \text { (2) }
\end{aligned}
$$

Therefore, we maintain a list $L$ of neighboring triangles $\left\{t_{i}\right\}$ whose counters have $1 \leq c_{i} \leq 3$. When the sweep segment hits a new triangle $t_{j}$, we have $c_{j}=1$ and add $t_{j}$ into $L$; when a counter $c_{j}=3$, after processing the current segment we remove $t_{j}$ from $L$.

Given a skeleton point $p$, for a boundary triangle mesh with $N_{V}$ vertices, it takes $O\left(N_{T} \log N_{T}\right)$ to compute and sort all triangles following their segment angles. When we are sweeping a segment $p v_{i}$, if the size of the active triangle list $L$ is $m$, it takes $O(m)$ intersection-detecting operations. Therefore, the total complexity is $O\left(N_{T} \log N_{T}+N_{V} \cdot m\right)$. The incident triangle around a vertex $v_{i}$ is generally very small (i.e., $m<\log N_{T}$ ). Therefore, the algorithm finishes visibility detection of $p$ in $O\left(N_{T} \log N_{T}\right)$ time. On a skeleton containing $k$ nodes, it takes $O\left(k N_{T} \log N_{T}\right)$ precomputation time to compute the visible region for all nodes.

\section{B. Greedy and Optimal Guarding}

Once visibility information for all skeletal nodes is computed, we want to pick a minimum sized point set that can cover all boundary vertices. It can now be converted to a setcovering problem, which is also NP-complete [40]: Given the universe point set $V=\left\{v_{i}\right\}, i=1, \ldots, N_{V}$, and a family $S$ of subsets $S_{j}=\left\{s_{j, k}\right\}, s_{j, k} \in V, j=1, \ldots, N_{S}$, a cover is a subfamily $C \subset S$ of sets whose union is $V$. We want to find a cover $C$ that uses the fewest subsets in $S$. Here, $V$ corresponds to the set of all vertices of $\partial M$; for each skeletal node $p_{j}, j=1, \ldots, N_{S}, S_{j}$ contains all boundary vertices visible to $p_{j}$. Each $C$ indicates a subset of skeletal nodes that can guard the entire region. Skeletons generated using medial-axis-based methods with dense enough nodes usually ensure that $S$ itself is a covering. This holds in all of our experiments. However, if a coarsely sampled skeleton cannot cover the entire $V$, we can easily include all those invisible vertices, i.e., their visible regions, into $S$.

A greedy strategy for the set covering is to iteratively pick the skeletal nodes $p$ that can cover the largest number of unguarded vertices in $V$, then remove all guarded vertices $v \in S(p)$ from $V$, meanwhile, and update $S$ accordingly since the universe becomes smaller, until $V=\emptyset$. The greedy strategy is effective, and it yields $O(\log n)$ approximation [41] to the set-covering problem.

An optimal selection can be computed by $0-1$ programming, also called integer linear programming (ILP). For every skeleton point $p_{i}, i=1, \ldots, N_{S}$, we assign a variable $x_{i}$ such that

$$
x_{i}= \begin{cases}1, & \text { if } p_{i} \text { is chosen } \\ 0, & \text { otherwise }\end{cases}
$$

The objective function to minimize is then $\sum_{i=1}^{N_{S}} x_{i}$, as we want to pick the fewest necessary points. Since $\forall v \in V$ should be covered, for each such $v_{i}$, at least one of its visible skeletal nodes $P_{i}=\left\{p_{j} \mid v_{i} \in S\left(p_{j}\right)\right\}$ should be picked. Therefore, we $\operatorname{minimize} \sum_{i=1}^{N_{S}} x_{i}$, subject to

$$
x_{i}=\{0,1\} \text {, and } \sum_{p_{j} \in P_{i}} x_{j} \geq 1 \quad \forall v_{i} \in V .
$$

This objective function can be minimized using branch-andbound algorithms. When the dimension is small (e.g., a few hundreds to a few thousands), we can use the TomLab optimization package [42] to solve it efficiently.

\section{Hierarchical Guarding}

Computing the optimal guarding based on ILP is highly time consuming and it limits the size of problems that we can handle: General 3-D volumetric shapes can easily have a number of vertices (20 000-200 000) on its boundary surface, which is too large for this optimization. On the other hand, the greedy algorithm generates the guards in a locally optimal manner. Furthermore, the greedy strategy is not robust against local geometric perturbations. For example, a small bump could lead to global structural variance of the guarding points. We propose a hierarchical guarding computation framework which is based on the progressive mesh [43], combining the $0-1$ programming optimization and the adaptive greedy refinement.

We simplify the boundary mesh $\partial M$ into several resolutions $\partial M^{i}=\left\{V^{i}, F^{i}\right\}, i=0, \ldots, m$, using a progressive mesh [43]. In the coarsest level $i=m$, ILP optimization is performed on all elements $v \in \partial M^{m}$, and we get the coarsest level guard set $G^{i}=\left\{g_{k}^{i}\right\}$. Then, we progress to $i=m-1$ level $\partial M^{m-1}=$ $\left(V^{m-1}, F^{m-1}\right)$.

1) Map existing guards $G^{i+1}=\left\{g_{k}\right\}$ to closest finer level skeletal nodes $G^{i}=\left\{g_{k}^{\prime}\right\}$ to locally adjust them to maximize their visible region $S\left(g_{k}^{\prime}\right)$.

2) Remove least significant guards $\left\{g|| S(g) \mid<\varepsilon N_{V}\right\}$ from $G^{i}$.

3) Remove covered vertices $\left\{v \mid v \in S(g), g \in G^{i}\right\}$.

Then, we solve ILP again on uncovered boundary vertices. With details increase in finer levels, new guards will be inserted into $G^{i}$. Before applying the ILP optimization, we further conduct four reduction (see in the following) operations on uncovered regions to reduce the dimensions of the optimization. This progressive refinement ends when all boundary vertices are covered on the finest level $i=0$.

Reduction: The dimension of the ILP optimization on each level can be reduced using the following reduction rules, without changing the size of the optimal solution. Suppose we store the visibility information in an incidence matrix $A$. If the skeletal node $p_{i}$ can see the vertex $v_{j}$, then we let $a_{i j}=1$, otherwise ( $\pi$ cannot see $v_{j}$ ), let $a_{i j}=0$. Originally the dimension of $A$ is $N_{S} \times N_{V}$. The following four rules are applied to reduce it.

1) If column $j$ has only one nonzero element at row $i$, we must take $p_{i}$ in order to see $v_{j}$. Therefore, add $p_{i}$ into $G$, and remove column $j$. In addition, for all nonzero element $a_{i k}$, remove column $k$ (we take $p_{i}$ : all points that it sees are guaranteed to be covered, and, thus, now can be removed).

2) If row $i_{1}$ has all its nonzero elements nonzero in row $i_{2}$, i.e., $a_{i_{1}, j}=1 \rightarrow a_{i_{2}, j}=1$, then $p_{i_{2}}$ sees all vertices that $p_{i_{1}}$ can see, and we can remove the entire row $i_{1}$. 


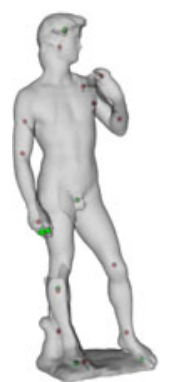

(a)

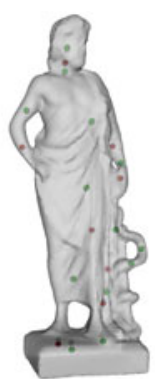

(b)

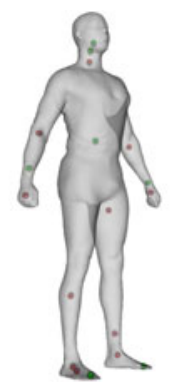

(c)

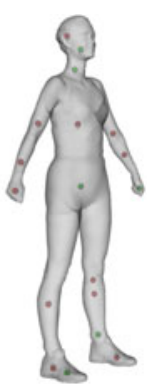

(d)
Fig. 3. Guarding statues using HILP. From left to right: Guarding on a few sculpture datasets. (a) Michelangelo's David. (b) Greek. (c) Cyberware Male. (d) Female. Small nodes are the guards, where green nodes are the latest computed guards on the finest level.

3) If column $j_{1}$ has all its nonzero elements nonzero in column $j_{2}$, i.e., $a_{i, j_{1}}=1 \rightarrow a_{i, j_{2}}=1$, then guarding $v_{j_{1}}$ guarantees the guarding of $v_{j_{2}}$, and we can remove the entire column $j_{2}$.

4) If the matrix $A$ is composed of several blocks, we partition $A$ to several small matrixes $\left\{A_{k}\right\}$.

In step 4, after removing vertices that have been seen by the adjusted guards from a coarser level, remaining boundary vertices could be partitioned to several connected components far away from each other, which can be optimized separately and more efficiently.

In our experiments, we simplify the boundary mesh to the coarsest level with 5000 vertices for the first round ILP optimization. Generally, we make each iteration to add in another 10000 vertices. When the size of constraints is around 5000, and the size of variables (skeletal nodes) is around 1000, the optimization usually takes $10-50 \mathrm{~s}$ to solve.

Our hierarchical scheme together with the reduction processing has the following important advantages over both the pure greedy strategy and the pure 0-1 optimization.

1) It is much faster than the nonlinear ILP optimization. The current framework can handle large-size geometric shapes.

2) With similar performance, it usually provides better guarding solutions than a pure greedy strategy.

3) It is hierarchical and, therefore, is robust and stable against geometric noise. In our HILP framework, refined local details tend to not change the global structure of the previously optimized guarding graph in coarser levels.

Fig. 3 shows some examples of HILP guarding on sculpture data, and Table II shows the runtime statistics. We use these irregular sculpture data to demonstrate the significant efficacy of our algorithm since our prototype pipelines on hand are relatively simple. To thoroughly cover and inspect complex pipeline system such as in Fig. 2, using HILP we can find its guarding point set efficiently.

\section{Autonomous Pipeline InSPECTION}

Our guarding algorithm computes the set of necessary checking spots for thorough inspection. The inspection robot only
TABLE II

GUARDING STATISTICS TABLE

\begin{tabular}{c|ccc|ccc}
\hline Models $\left(N_{V}\right)$ & $N_{I}$ & $N_{G}$ & $N_{H}$ & $t_{I}$ & $t_{G}$ & $t_{P}$ \\
\hline Armadillo $(20,002)$ & - & 38 & 30 & - & 590.8 & 601.4 \\
Female (10,002) & 13 & 18 & 14 & $2,046.2$ & 279.1 & 300.3 \\
Greek $(9,994)$ & 15 & 22 & 18 & $4,122.4$ & 307.4 & 312.9 \\
David $(9,996)$ & 16 & 22 & 17 & $107,391.2$ & 245.1 & 248.2 \\
Michael $(20,002)$ & - & 46 & 31 & - & 333.0 & 342.9 \\
Gorilla $(30,004)$ & - & 60 & 46 & - & 482.8 & 498.2 \\
\hline
\end{tabular}

$N_{V}$ is the boundary surface vertex number; $N_{I}, N_{G}, N_{P}$ indicate the number of necessary guards computed by ILP, greedy, and PILP approaches, respectively; $t$ shows the computational time in seconds.

Guarding of big models cannot be solved directly using ILP, so their statistics are nor applicable.

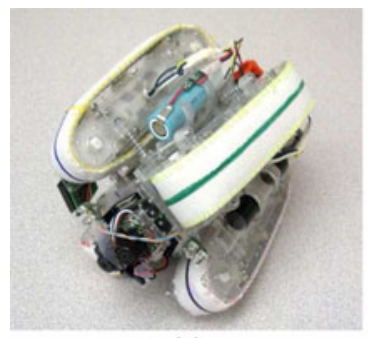

(a)

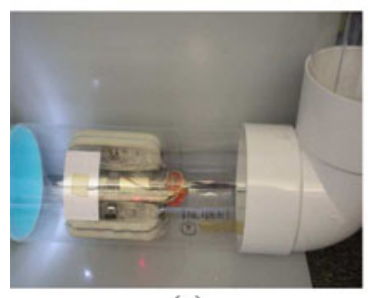

(c)

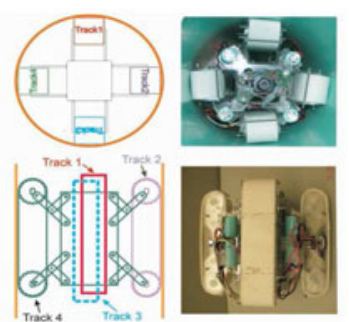

(b)

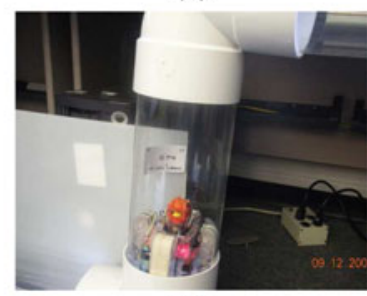

(d)
Fig. 4. Prototype robot FAMPER. (a) and (b) Robot and its design. (c) and (d) Robot inspecting a pipe.
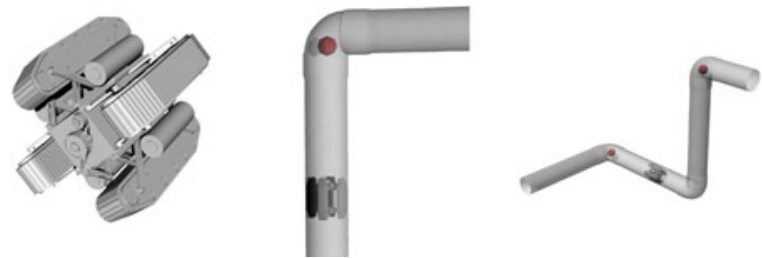

Fig. 5. Simulation environment. The 3-D model of the FAMPHER robot in the pipeline; red points denote guarding points.

needs to go to each guarding point, construct the current height maps (see Section IV-A), and compare them with the precalculated templates for abnormal identifications. When geometric changes are detected, the system refines the identification of the abnormal areas (see Section IV-B), and extract the boundary of the damaged region (see Section IV-C).

\section{A. Height Maps Acquisition}

The geometry of the pipeline environment is measured using laser range finders in our system. The distance from the robot to a point on the wall is captured and stored. A range image stores a set of depth information in a rectangle viewport along a direction, and we call it a height map. 


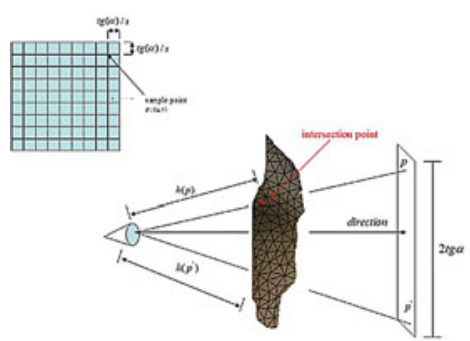

(a)

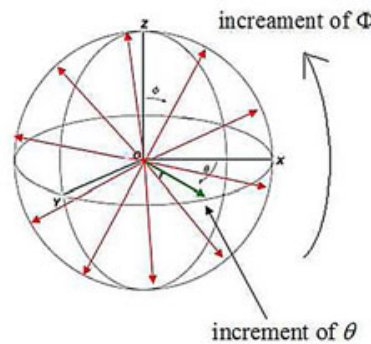

(b)
Fig. 6. (a) Shot of the laser scanning. The sample grid density is decided by the sample rate and the scan range of the laser scanner. Every sample point has a planar coordinate $(u, v)$ corresponding to the actual intersection point in 3-D space. The laser scanner rotates to a direction and does a snapshot to project the surface to a planar with a range of $2 \tan \alpha$. The red point indicates the intersection result of $p$ in the 3-D region, and the corresponding height data $h(p)$ are stored for detection. Scanning at every sample point, the height map is constructed. (b) Motion plan of the scanner. The scanner rotates to cover the whole spherical area. The red arrowheads are the rotated direction of $\phi$, and the green is one step of rotation increment of $\theta$.

The abnormal detection is based on the comparison between precalculated height map templates and the current height maps. We compare height maps on each inspection point. Suppose the laser scanning has two parameters: the scan range angle $\alpha$ and the sampling rate $s . \alpha$ decides the field of view of the scanner, and $s$ indicates the sampling resolution inside the field of view. As Fig. 6(a) shows, given a shooting direction, the scanner takes a snapshot of the environment, which produces a height map on a planar square region $R$, uniformly sampled with $s \times s$ points $P$. Since one snapshot can only cover an area within the current view angle, a planed motion sequence is necessary for the laser scanner to rotate and take pictures to cover the entire $360^{\circ}$. The laser scanner with the scan range $\alpha$ and the sample rate $s$ is placed at a guarding position $o$, which points toward an initial direction $L$; the height map acquisition processes can be simply conducted as follows.

1) Using the local spherical coordinate system which is defined at $o$, given a direction $L(r, \phi, \theta), L$ takes a snapshot and gets a depth image $P(\alpha, s)$ [see Fig. 6(a)]. A depth is defined on every point on the image $p \in P$, whose 2-D coordinate can be defined as $(u, v), 0 \leq u \leq 1,0 \leq v \leq 1$.

2) 3-D position of each boundary point $v$ can be derived from the depth on its projection $p(u, v)$. The transformation can be represented by a rotation matrix.

3) After taking one depth image, the laser scanner rotates for another height map. The motion plan of the scanner follows the rotation sequences: First fix $\phi$, rotate $\theta$ for $N$ times, increase $\pi / \alpha$ iteratively, and then increase $\phi$ to perform the $\theta$ rotation again until the whole spherical region is covered. The rotation path is shown in Fig. 6(b).

4) Finally, we get the set of height maps $\left\{H_{L}\right\}$ collected in the aforementioned steps and save them together with the starting projection direction on every guarding point.

In practice, during height map acquisition, the geometry of regions far away from a guard can be visible but captured less accurately because of the precision of the range finder or the

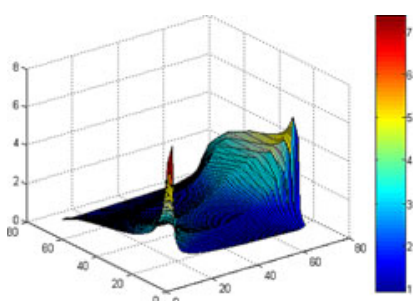

(a)

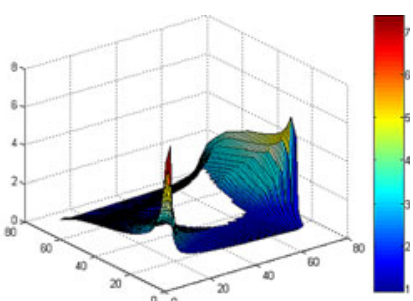

(b)
Fig. 7. Height maps comparison. (a) Template height map. (b) Height map of an abnormal region. The missing region indicates a hole.

sampling resolution. To tackle this issue, before guarding computation, we add a simple parameter $d_{p}$ for each skeleton node $p$ : If a boundary point $q \in \partial M$ is visible to $p$ but far away, i.e., distance $|\overline{p q}|>d_{p}$, we consider $q$ to be invisible. In other words, a guard only sees points in a bounded distance. The whole optimization algorithm can be applied exactly in the same way. In long and thin environments, more guards may be necessary, but each guarding region will have less long antenna, and the inspection accuracy will be improved. A heuristic setting for $d_{p}$ can be $d_{p}=\alpha \cdot d_{p}^{0}$, where $\alpha$ is a constant parameter, and $d_{p}^{0}$ is the distance from $p$ to its nearest visible boundary point.

\section{B. Abnormal Boundary Detection}

After all height maps are obtained, on every guarding point, we compare the precollected template height maps $\left\{H_{L}\right\}$ [see Fig. 7(a)] and the current height maps $\left\{H_{L}^{\prime}\right\}$ [see Fig. 7(b)]. If the height information changes $\left|h(u, v)-h^{\prime}(u, v)\right|>\varepsilon$, we consider the region around $(u, v)$ as a defective region, and report $(u, v)$ as a abnormal point. $\varepsilon$ is a similarity threshold. In practice, the acquired depth data could have geometric and topological noise. By adjusting $\varepsilon$, the system can tolerate small deviations because of certain acquisition noise. On the other hand, a local efficient data preprocessing step in topological denoise or geometric completion/fairing [44], [45] could also be helpful in cleaning environment noise.

Compared with reporting simply a set of sampled abnormal points, an accurate estimation of the bad region's shape is desirable. When a defective region is detected, in order to "zoom in" to see the shape of the defective region, we need to examine more sampling points by performing denser scanning around this region. During the initial scanning of the original pipeline, we may not do very dense sampling; therefore, with the same number of depth acquisition, we can capture a larger region in every shot for better efficiency. Regions among sampling points are approximated using bilinear interpolation $h(E)$ $\approx \frac{h\left(u_{1}, v_{1}\right)}{\left(u_{2}-u_{1}\right)\left(v_{2}-v_{1}\right)}\left(u_{2}-u\right)\left(v_{2}-v\right)+\frac{h\left(u_{2}, v_{1}\right)}{\left(u_{2}-u_{1}\right)\left(v_{2}-v_{1}\right)}\left(u-u_{1}\right)$ $\left(v_{2}-v\right)+\frac{h\left(u_{1}, v_{2}\right)}{\left(u_{2}-u_{1}\right)\left(v_{2}-v_{1}\right)}\left(u_{2}-u\right)\left(v-v_{1}\right)+\frac{h\left(u_{2}, v_{2}\right)}{\left(u_{2}-u_{1}\right)\left(v_{2}-v_{1}\right)}$ $\left(u-u_{1}\right)\left(v-v_{1}\right)$.

To report a refined boundary shape of an abnormal region, we use a quadtree [46] subdivision scheme: Near such a region, boundary cells split accordingly to get the refined geometry. For each new point $\left(u^{\prime}, v^{\prime}\right)$, we detect its height $h^{\prime}\left(u^{\prime}, v^{\prime}\right)$ and compare it with the interpolated height on the template 


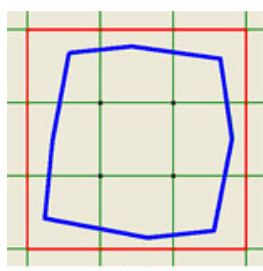

(a)

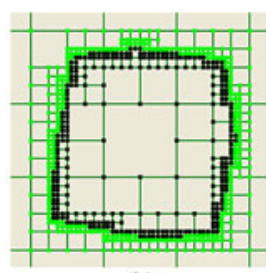

(b)

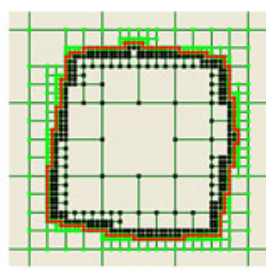

(c)
Fig. 8. Quadtree splitting and boundary extraction. (a) Polygon colored in blue indicates a defective region on the pipe wall. The green nodes are normal points, and the black ones indicate the abnormal height data; the boundary extracted from the quadtree without quadtree splitting, colored in red, is coarse (b) Quadtree cells split. (c) Extracted boundary (red curves) after quadtree refinement.

height map $h\left(u^{\prime}, v^{\prime}\right)$. Fig. 7 shows an example. The max level of the subdivision is determined by the resolution of the laser range finder. The boundary of holes, clogs, and deformations can be detected/refined using this paradigm, since these geometric changes always lead to changed height maps.

\section{Boundary Extraction}

When the refinement is done, the boundary of the abnormal region can be extracted and reported. We conservatively link the normal points on boundary cell up to form the boundary: Starting from a normal point on a boundary cell, linking the normal points in the cell along the edge, getting a neighboring boundary cell, and repeating this process until all boundary cells are traversed.

Fig. 8 illustrates the process of quadtree splitting and extraction. In a snapshot, the grids are the cells, and the green polygon indicates a hole on the pipeline wall. Normal points are colored in green, while abnormal points are colored in black. In the coarsest resolution, we get the red boundary shown in (a). We can get finer boundary shape (b), and the extracted boundary is depicted in red in (c).

\section{RESULTS OF SIMULATED EXPERIMENTS}

Our guarding-driven pipe inspection system can be implemented on the prototype pipeline robot [2], which can be used for the inspection of pipelines. This robot consists of four wallpress caterpillars that are operated by two dc motors each to provide steering capability to go through $45^{\circ}$ elbows, $90^{\circ}$ elbows, T-branches, and Y-branches, and make a superior performance in all types of complex networks of pipelines (see Fig. 4). The robot is also equipped with a powerful computing system that makes it extendable to various sensing and actuating devices, such as in our experimental system, for localization and laser scanning. The height information can be obtained using a multislit laser range scanner [47], which has the size of about $110 \mathrm{~m}$ $\times 90 \mathrm{~mm}$ and includes a laser projector and a charge-coupled device (CCD) camera (the laser projector in [47] is StockerYale Mini-715L, which projects 15 slits, with an adjacent slit angle $2.3^{\circ}$; the CCD camera is Point Grey Research Flea2, which measures 330 points). The height information can also be ob- tained by laser scanners in [21] and [34] or other range cameras (e.g., [48] and [49]).

The rotation of the sensor can be controlled by a small mechanical platform installed in the rear part of the robot; therefore, the scan can be conducted radially inside the pipe toward different directions. Several effective sensing platforms with similar mechanism have been developed [21].

We develop a simulated platform for testing our algorithm (see Fig. 5), which simulates the process of inspection. We apply our procedure on this platform using several complicated 3 -D virtual pipelines. The simulated results are convincing, and shows the effective inspection on pipeline geometry.

\section{A. Hole Detection}

If a hole appears on the pipeline, it can be identified online when the robot reaches the guarding point that covers this region, and matches the captured range depth images with the stored templates. We simulate this on pipeline meshes $M$ by randomly generating some missing regions. An experiment is shown in Fig. 9. A pipeline model and the necessary guards are shown in (a), where regions covered by each guard are rendered in a specific color for the visualization purpose. Any given region of the pipeline is covered by at least one guard and, therefore, is colorized. The height maps can then be generated as templates, measuring the "correct" distance from each guarding site to the pipe wall toward specific directions. This simulates range images obtained by a laser scanner. Now, we simulate the appearance of defected regions on the pipeline by generating some missing regions as shown in (b). When the robot checks height maps on guarding points, these holes can be immediately detected and illustrated in (c).

Another example is shown in Fig. 10; this pipe is guarded by 12 points (a). In addition, the damaged region of the pipe is big and with complex topology (b). In this case, the robot should check from more than one guarding points in order to detect the entire shape of such a big hole. The entire defect geometry is extracted by composing boundaries detected from different guarding sites. The merged boundary loop is illustrated in (c).

\section{B. Deformation Detection}

Small deformation such as bending, erosion, etc., can also be detected in our system as shown in Fig. 11. The detected deformed region is colored in red.

\section{Clogging Detection}

Clogging also changes the scanned geometry of the pipeline and can be detected. Fig. 12 shows an example. The clogged solid (green) is detected, and its boundary geometry is reconstructed using height maps as illustrated in dark red. The robot will report the clogs when it is detected. In this example, the reconstruction merges the geometry of the blocking stuff from two aspects (from two guarding points) using their corresponding height maps. 


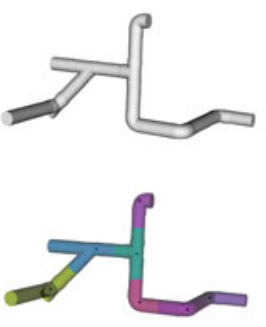

(a)

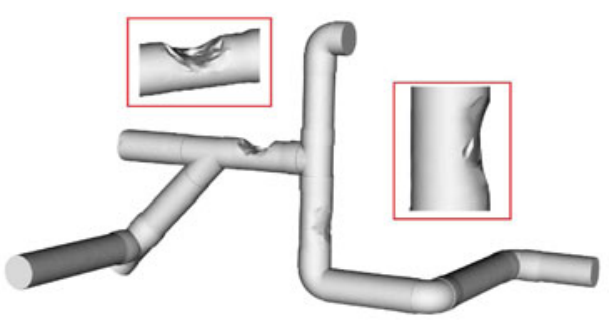

(b)

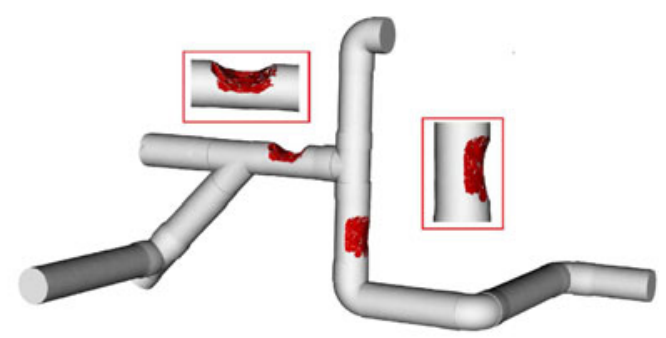

(c)

Fig. 9. Inspection process on a simulated pipeline. (a) (Upper) Simulated model. (Lower) Regions guarded by different guard points encoded in different colors. (b) Damaged pipe with some holes. (c) Damages are detected, whose boundaries are extracted and shown in green.

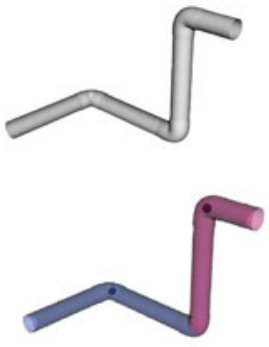

(a)

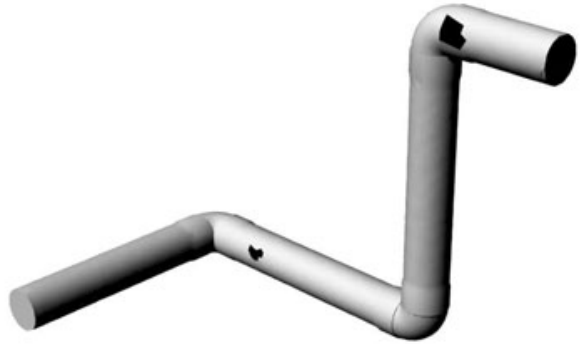

(b)

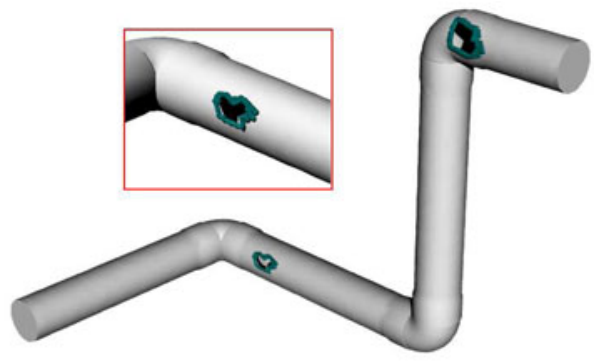

(c)

Fig. 10. Guarding and inspection process on a more complicated pipeline. (a) (Upper) Simulated model. (Lower) Guarding points and guarded regions rendered

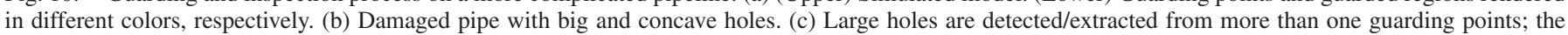
whole big boundary is composed of several extracted subboundaries and identified separately from different guarding points, as shown in green.

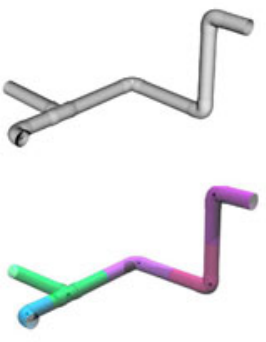

(a)

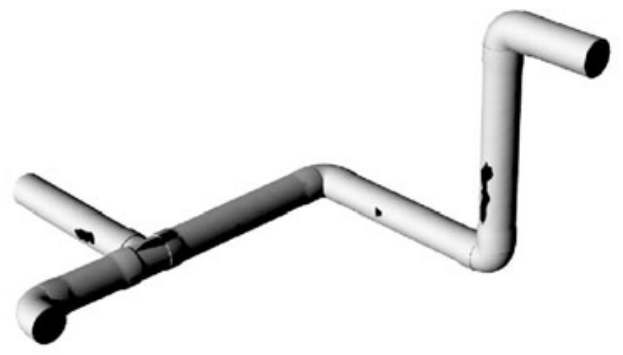

(b)

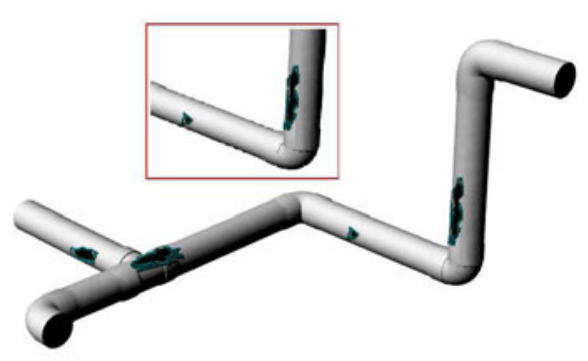

(c)

Fig. 11. Inspection process on a deformed pipeline. (a) (Upper) Simulated model. (Lower) Regions guarded by different guard points encoded in different colors. (b) Deformed pipe. (c) Deformations are detected, whose regions are extracted (and refined) and shown in red.
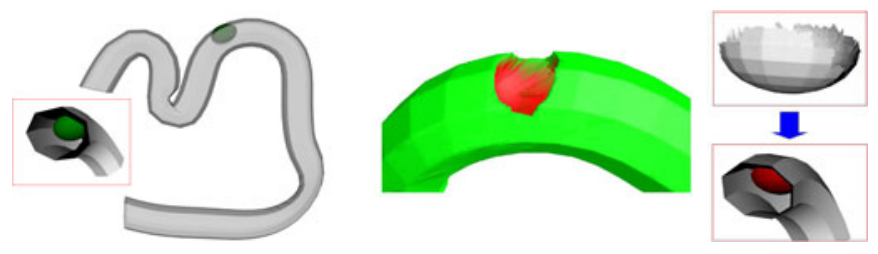

Fig. 12. (Left) Pipeline blocked by a solid (in green). (Middle) Clog detection on the pipeline. Height maps constructed and merged by detected views from different guarding points. Green regions indicate normal status, while the red regions indicate the abnormal height information corresponding to the clog geometry. (Right) Inspection result, which is the reconstructed surface colored in dark red, describes the location and geometry of the blocking region.

\section{CONCLUSION}

We have proposed an efficient 3-D guarding algorithm that can cover a given complicated environment using as few as pos- sible points. Finding an efficient solution to the fundamental problem of how to inspect on fewest points yet thoroughly covering the entire environment can greatly benefit the autonomous design of inspection and exploration robots.

We have developed a simulation system of pipeline inspection, and conducted experiments to evaluate the efficacy of our system. With our optimal guarding, abnormal geometric changes of the pipeline such as holes, clogs, and deformation can be thoroughly detected online.

The remaining challenging issue for the current system is the dynamic environment mapping. The initial geometry of the pipeline system needs to be scanned to a digital model before the region-guarding computation. In addition, after the guarding spots are computed, the pipeline wall needs to be marked so that the robot can localize itself to know whether it is on the spot. The development of a system that a robot without this prior knowledge can do simultaneous 3-D mapping (i.e., reconstructing 
the map of the environment) and localization will be highly desirable. In the near future, we will study these localization and dynamic environment mapping problems by exploring effective partial matching of range images.

\section{ACKNOWLEDGMENT}

This paper highlights the autonomous robotic systems' dynamical problems associated with technology and engineering sciences. A connecting link between these two is that computer science plays an important role in both fields. This concept is inspired by Prof. Hopcroft's paper titled "the impact of robotics on computer science" [50] published in the 1990s.

\section{REFERENCES}

[1] Hogeschoolzeeland. (2007). Pipes, tubes, machinery and steam turbine at a power plant [Online]. Available http://goo.gl/fRp3c

[2] J.-H. Kim, G. Sharma, and S. Iyengar, "FAMPER: A fully autonomous mobile robot for pipeline exploration," in Proc. IEEE Int. Conf. Ind. Technol., 2010, pp. 517-523.

[3] B. Ben-Moshe, M. J. Katz, and J. S. Mitchell, "A constant-factor approximation algorithm for optimal terrain guarding," in Proc. ACM/SIAM Symp. Discr. Algorithms, 2005, pp. 515-524.

[4] V. Chvatal, "A combinatorial theorem in plane geometry," J. Combinatorial Theory, vol. 18, pp. 39-41, 1975.

[5] J. O. Rourke, "Galleries need fewer mobile guards: A variation on Chvatal's theorem," Geometriae Dedicata, vol. 14, pp. 273-283, 1983.

[6] J. Urrutia, "Art gallery and illumination problems," in Handbook of Computational Geometry, Amsterdam, The Netherlands: North-Holland, 2000, pp. 973-1027.

[7] J. Kahn, M. Klawe, and D. Kleitman, "Traditional galleries require fewer watchmen," SIAM J. Algebr. Discrete Methods, vol. 4, no. 2, pp. 194-206, 1983.

[8] J. O. Rourke, "An alternate proof of the rectilinear art gallery theorem," J. Geometry, vol. 21, pp. 118-130, 1983.

[9] J. O'Rourke, Art Gallery Theorems and Algorithms. London, U.K.: Oxford Univ. Press, 1987.

[10] F. Hoffmann, M. Kaufmann, and K. Kriegel, "The art gallery theorem for polygons with holes," in Proc. 32nd Annu. Symp. Found. Comput. Sci. IEEE Comput. Soc., 1991, pp. 39-48.

[11] I. Bjorling-Sachs and D. Souvaine, "An efficient algorithm for guard placement in polygons with holes," Discr.Comput.Geom., vol. 13, pp. 77 109, 1995.

[12] E. Gyori, F. Hoffmann, K. Kriegel, and T. Shermer, "Generalized guarding and partitioning for rectilinear polygons," Comput. Geom., vol. 6, no. 1, pp. 21-44, 1996.

[13] S. Fisk, "A short proof of Chvatal's watchman theorem," J. Combinatorial Theory, Series B, vol. 24, no. 3, p. 374, 1978.

[14] D. Avis and G. Toussaint, "An efficient algorithm for decomposing a polygon into star-shaped polygons," Pattern Recognit., vol. 13, pp. 395398, 1981.

[15] J. O'Rourke and K. Supowit, "Some NP-hard polygon decomposition problems," vol. 29, pp. 181-190, 1983.

[16] D. T. Lee and A. K. Lin, "Computational complexity of art gallery problems," IEEE Trans. Inf. Theory, vol. 32, no. 2, pp. 276-282, Mar. 1986.

[17] D. Schuchardt and H.-D. Hecker, "Two np-hard art-gallery problems for ortho-polygons," Math. Logic. Quart., vol. 41, pp. 261-267, 1995.

[18] M. J. Katz and G. S. Roisman, "On guarding the vertices of rectilinear domains," Comput. Geom.: Theory Appl., vol. 39, no. 3, pp. 219-228, 2008.

[19] A. Efrat and S. Har-Peled, "Guarding galleries and terrains," Inf. Process. Lett., vol. 100, no. 6, pp. 238-245, 2006.

[20] J.-M. Lien, "Approximate star-shaped decomposition of point set data," presented at the Eurograph. Symp. Point Based Graph., Prague, Czech Republic, 2007.

[21] A. Nassiraei, Y. Kawamura, A. Ahrary, Y. Mikuriya, and K. Ishii, "Concept and design of a fully autonomous sewer pipe inspection mobile robot "KANTARO,"” in Proc. IEEE Int. Conf. Robot. Autom., Apr. 10-14, 2007, pp. 136-143.
[22] J. Y. Choi, H. Lim, and B.-J. Yi, "Semi-automatic pipeline inspection robot systems," in Proc. SICE-ICASE Int. Joint Conf., 2006, pp. 2266-2269.

[23] S. Costello, D. Chapman, C. Rogers, and N. Metje, "Underground asset location and condition assessment technologies," Tunnelling Underground Space Technol., vol. 22, nos. 5-6, pp. 524-542, 2007.

[24] J.-H. Kim, G. Sharma, N. Boudriga, and S. Iyengar, "Ramp system for proactive pipeline monitoring," in Proc. Int. Conf. Commun. Syst. Netw., 2010, pp. 1-2.

[25] J. Kim, G. Sharma, N. Boudriga, and S. Iyengar, "Spamms: A sensor-based pipeline autonomous monitoring and maintenance system," in Proc. Int. Conf. Commun. Syst. Netw., 2010, pp. 1-10.

[26] R. Wirahadikusumah, D. M. Abraham, T. Iseley, and R. K. Prasanth, "Assessment technologies for sewer system rehabilitation," Autom. Construct., vol. 7, no. 4, pp. 259-270, 1998.

[27] D.-H. Koo and S. T. Ariaratnam, "Innovative method for assessment of underground sewer pipe condition," Autom. Construct., vol. 15, no. 4, pp. 479-488, 2006

[28] O. Duran, K. Althoefer, and L. Seneviratne, "State of the art in sensor technologies for sewer inspection," IEEE Sens. J, vol. 2, no. 2, pp. 73-81, Apr. 2002.

[29] W. W. Zhang and B. H. Zhuang, "Non-contact laser inspection for the inner wall surface of a pipe," Meas. Sci. Technol., vol. 9, no. 9, p. 1380, 1998.

[30] J. Moraleda, A. Ollero, and M. Orte, "A robotic system for internal inspection of water pipelines," IEEE Robot. Autom. Mag., vol. 6, no. 3, pp. 30-41, Sep. 1999.

[31] M. Silk, "The determination of crack penetration using ultrasonic surface waves," NDT Int., vol. 9, no. 6, pp. 290-297, 1976.

[32] D. Levesque, M. Ochiai, A. Blouin, R. Talbot, A. Fukumoto, and J.-P. Monchalin, "Laser-ultrasonic inspection of surface-breaking tight cracks in metals using SAFT processing," in Proc. IEEE Ultrason. Symp., Oct. 8-11, 2002, vol. 1, pp. 753-756.

[33] J. Hertzberg and F. Kirchner, "Landmark-based autonomous navigation in sewerage pipes," in Proc. 1st Euromicro Workshop Adv. Mobile Robot, Oct. 1996, pp. 68-73.

[34] H. Streich and O. Adria, "Software approach for the autonomous inspection robot MAKRO," in Proc. IEEE Int. Conf. Robot. Autom., Apr. 2004, vol. 4, pp. 3411-3416.

[35] J. M. Mirats Tur and W. Garthwaite, "Robotic devices for water main in-pipe inspection: A survey," J. Field Robot., vol. 27, pp. 491-508, Jul./Aug. 2010.

[36] T. He, L. Hong, D. Chen, and Z. Liang, "Reliable path for virtual endoscopy: Ensuring complete examination of human organs," IEEE Trans. Vis. Comput. Graphics, vol. 7, no. 4, pp. 333-342, Oct.-Dec. 2001.

[37] D.-G. Kang and J. B. Ra, "A new path planning algorithm for maximizing visibility in computed tomography colonography," IEEE Trans. Med. Imag., vol. 24, no. 8, pp. 957-968, Aug. 2005.

[38] N. Cornea, D. Silver, and P. Min, "Curve-skeleton properties, applications, and algorithms," IEEE Trans. Vis. Comput. Graphics, vol. 13, no. 3, pp. 530-548, May 2007.

[39] T. K. Dey and J. Sun, "Defining and computing curve-skeletons with medial geodesic function," in Proc. 4th Eurographics Symp. Geometry Process., Eurographics Assoc. Aire-la-Ville, Switzerland, 2006, pp. $143-$ 152.

[40] J. Kahn, M. Klawe, and D. Kleitman, "Traditional galleries require fewer watchmen," SIAM J. Algebraic Discr. Methods, vol. 4, no. 2, pp. 194-206, 1983.

[41] D. S. Johnson, "Approximation algorithms for combinatorial problems," in Proc. 5th Annu. ACM Symp. Theory Comput., New York, 1973, pp. 3849.

[42] “TOMLAB v3.0 User's Guide," Dep. Math. Phys., Malardalen Univ., Västerås, Sweden, Tech. Rep. IMa-TOM-2001-01, 2001.

[43] H. Hoppe, "Progressive meshes," in Proc. SIGGRAPH., New York, 1996, pp. 99-108.

[44] H. Schoner, B. Moser, A. A. Dorrington, A. D. Payne, M. J. Cree, B. Heise, and F. Bauer, "A clustering based denoising technique for range images of time of flight cameras," in Proc. Int. Conf. Comput. Intell.Model., Control Autom., 2008, pp. 999-1004.

[45] X. Li, Z. Yin, L. Wei, S. Wan, W. Yu, and M. Li, "Symmetry and template guided completion of damaged skulls," Comput. Graph., vol. 35, pp. 885893, Aug. 2011.

[46] R. Finkel and J. Bentley, "Quad trees: A data structure for retrieval on composite keys," Acta Informatica, vol. 4, pp. 1-9, 1974.

[47] T. Kuroki, K. Terabayashi, and K. Umeda, "Construction of a compact range image sensor using multi-slit laser projector and obstacle detection 
of a humanoid with the sensor," in Proc. IEEE/RSJ Int. Conf. Intell. Robots Syst., Oct. 2010, pp. 5972-5977.

[48] J. Thielemann, G. Breivik, and A. Berge, "Pipeline landmark detection for autonomous robot navigation using time-of-flight imagery," in Proc. IEEE Comput. Soc. Conf. Comput. Vis. Pattern Recognit. Workshops, Jun. 23-28, 2008, pp. 1-7.

[49] J. Horn and J. Russ, "Localization of a mobile robot by matching 3d-laserrange-images and predicted sensor images," in Proc. Intell. Veh. Symp., Oct. 24-26, 1994, pp. 345-350.

[50] J. E. Hopcroft, "The impact of robotics on computer science," Commun. ACM, vol. 29, pp. 486-498, Jun. 1986.

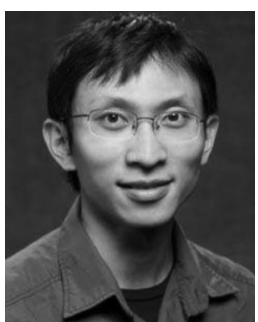

Xin $\mathbf{L i}$ (M'09) received the B.S. degree from the University of Science and Technology of China, Hefei, China, and the M.S. and Ph.D. degrees from Stony Brook University, Stony Brook, NY, all in computer science.

$\mathrm{He}$ is currently an Assistant Professor with the Department of Electrical and Computer Engineering, Center for Computational and Technology, Louisiana State University, Baton Rouge. His research interests include geometric computing/modeling, computer graphics, vision, and visualization.

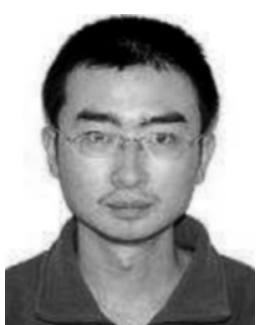

Wuyi Yu (S'11) received the B.E. degree in electrical engineering from Xiamen University, Xiamen, China, where he is currently working toward the Ph.D. degree with the Department of Automation

He is currently a Visiting Student with the Department of Electrical and Computer Engineering, Louisiana State University, Baton Rouge. His research interests include geometric modeling, computer graphics, shape analysis and decomposition, and volumetric mapping.

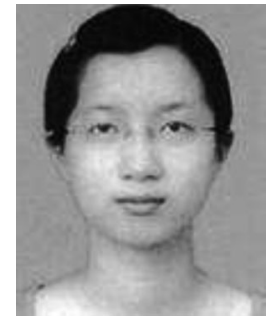

Xiao Lin received the B.E. degree in electrical engineering from Xiamen University, Xiamen, China, where she is currently working toward the Graduate's degree with the Department of Automation.

Her research interests include computer graphics, shape deformation and editing, and surface mapping.

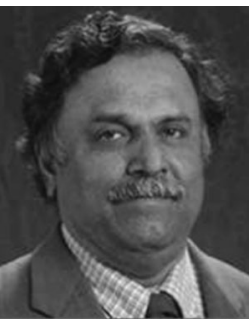

S. S. Iyengar (F'95) received the Ph.D. degree in computer science from Mississippi State University.

$\mathrm{He}$ is a Ryder Professor of Computer Science and the Director of the School of Computing Science and Information computing with Florida International University, Miami. His research interests include the design and analysis of high-performance algorithms, image analysis for medical applications, distributed sensor networks, parallel and distributed computing, and computational aspects of robotics applications.

Prof. Iyengar is a Fellow of the Association for Computing Machinery and the Advancing Science Serving Society and a member of the European Academy of Sciences. 